\title{
BLOCKCHAIN BASED CROSS ORGANIZATIONAL WORKFLOW MANAGEMENT SYSTEM
}

\author{
Hirofumi Nagano ${ }^{1}$, Taku Shimosawa ${ }^{1}$, Atsushi Shimamura ${ }^{2}$ and Norihisa Komoda ${ }^{3}$ \\ ${ }^{1}$ Research \& Development Division, Hitachi America, Ltd., 2535 Augustine Dr, Santa Clara, California, United States \\ ${ }^{2}$ Research \& Development Group, Hitachi Ltd., 1-280, Higashi-Koigakubo, Kokubunji-shi, Tokyo, Japan \\ ${ }^{3}$ Code Solutions Co., Ltd., 1-2-11-9F, Edobori, Nishi-ku, Osaka, Japan
}

\begin{abstract}
Currently most of the workflows in enterprise business processes are implemented and managed as information system. However, workflows between organizations are still processed manually based on paper document because it is difficult for the organizations to trust the process and the result of workflow in the system which is provided by one of the organizations or a third-party organization. In this paper, a system architecture for cross organizational workflow management utilizing blockchain technology is proposed. It eliminates every Single Point of Trust (SPoT) which causes falsification risk of the process and data. Our proposed system architecture accommodates two types of smart contracts for workflow definition and workflow processing, which validates related data in every transaction. By this architecture, each organization can be aware of the data falsification when the data on one of the organization nodes is falsified by a malicious workflow administrator or a system administrator. Through evaluation of the falsification risks based on the attack scenarios along workflow life cycle, it is confirmed that all the risks are avoided by the proposed system architecture.
\end{abstract}

\section{KEYWORDS}

Business Process, Workflow, Blockchain, System Security

\section{INTRODUCTION}

Along with the improvement of information technology, business processes in enterprise have become to be managed by information systems. The method to describe and manage business process is called Business Process Management (BPM) and workflow management is one of the key function of BPM (van der Aalst, 2016), where remarkable progress has been made through application to practical use cases (Kumar \& Zhao, 2002). The benefit of workflow management system is faster processing with less human effort (Reijers, et al., 2016). However, workflows between organizations are still document-based and processed manually. This is because most of existing workflow management systems consist of a single workflow engine using a single database (Stohr \& Zhao, 2001) assuming internal use in an organization. There have been several attempts to take advantage of distributed processing technologies such as WWW, CORBA, XML and Java to integrate systems of different organizations (Stohr \& Zhao, 2001), but in order to secure the reliability of transactions between organizations which do not fully trust each other, it is imperative to provide immutable and reliable record of process and data of workflows among organizations.

Blockchain technology, which is originally introduced as a platform technology for Bitcoin (Nakamoto, 2008), presents a feature to provide immutable data store for multiple organizations that supports requirements of workflows between organizations. Focused on this feature, companies have been working on many Proof-of-Concepts to evaluate the feasibility of this technology in supply chain management, trade finance, and other business cases (Hileman \& Rauchs, 2017) (World Trade Organization, 2018) (World Economic Forum, 2018). As one of the applications of blockchain technology, some approaches to implement workflow management systems among multiple organizations are proposed. For instance, the authors in (Alves, 2020) propose an architecture to integrate Business Process Management System (BPMS) and blockchain platform in order to guarantee transparency and tamper-proof information among participating parties. However, the intercommunication between BPMS and blockchain or the centralized 
BPMS can be a Single Point of Trust (SPoT) which a malicious user can attack to falsify related data. Another approach is to design specific workflow by designing method or tool and implement it as a smart contract on blockchain to secure that the workflow is processed as defined among untrusted organizations (Fridgen, et al., 2018) (Weber, et al., 2016). However, workflow definition should be able to be modified dynamically based on changing business requirements of each organization and the workflow administrator should not be SPoT. Therefore, more holistic approach is required which securely manages whole process of workflow life cycle among trustless organizations.

In this paper, a system architecture of cross organizational workflow management which eliminates SPoT throughout the workflow lifecycle including defining, executing, and auditing process is proposed. This architecture avoids falsification risks of both process and data of workflow. Based on the implementation of the proposed system architecture, the security risks of the proposed system architecture are evaluated based on the attack scenarios along the workflow lifecycle and confirmed that the risks are avoided by the proposed architecture.

\section{REQUIREMENT FOR CROSS ORGANIZATIONAL WORKFLOW MANAGEMENT SYSTEM}

\subsection{Definition of Workflow}

This section describes the definition of workflow in this paper. Workflow is a part of enterprise business process and consists of several processes including creating, amending, rejecting, and approving a proposal among stakeholders to finally have an agreement on the proposal, while recording the whole process. In the workflow management system, there are four types of users as follows.

- Workflow administrators: Users who are responsible to define and maintain workflow definition

- Workflow processors: Users who initiate or process workflow

- Auditors: Users who audit the record of processed workflow

- System administrator: Users who are responsible to maintain the workflow management system

There are many patterns of workflow structure (van der Aalst, et al., 2003), but it is defined as a data structure as shown in Table 1. In this example, the workflow consists of five steps and the sequence of the workflow (Figure 1) is defined as a relationship between each steps defined in "precondition" and "next step" field.

Table 1. Example of workflow definition

\begin{tabular}{|c|c|c|c|c|}
\hline Step & Role & Operation & Precondition & Next step \\
\hline Initiate Request & Initiator & set "Item 1", "Item 2" & - & Update Request \\
\hline Update Request & Approver1 & $\begin{array}{l}\text { approve "Item 1", "Item 2" } \\
\text { set "Item 3", "Item 4" }\end{array}$ & Initiate Request & $\begin{array}{l}\text { Approve Request } 1 \\
\text { Approve Request } 2\end{array}$ \\
\hline Approve Request1 & Approver2 & $\begin{array}{l}\text { approve "Item 1", "Item 2", } \\
\text { "Item 3", "Item 4" }\end{array}$ & Update Request & Approve Request 3 \\
\hline Approve Request2 & Approver3 & $\begin{array}{l}\text { approve "Item 1", "Item 2", } \\
\text { "Item 3", "Item 4"" }\end{array}$ & Update Request & Approve Request 3 \\
\hline Approve Request3 & Apprver4 & $\begin{array}{l}\text { approve "Item 1", "Item 2", } \\
\text { "Item 3", "Item 4" }\end{array}$ & $\begin{array}{l}\text { Approve Request1 } \\
\text { Approve Request } 2\end{array}$ & \\
\hline & & & & \\
\hline InitiateReques & & st & & \\
\hline
\end{tabular}

Figure 1. Example of workflow sequence

When this workflow is processed, the transaction record should be recorded as Table 2 to know the status of the workflow and to be audited afterwards. 
Table 2. Example of transaction record

\begin{tabular}{llll}
\hline Date & Operator & Step & Action \\
\hline 8/9/2020 09:05 & AAA & Initiate Request & Initiated \\
8/9/2020 10:12 & BBB & Update Request & Approved \\
8/9/2020 11:23 & CCC & Approve Request1 & Approved \\
8/9/2020 13:41 & DDD & Approve Request2 & Rejected \\
\hline
\end{tabular}

The workflow management system provides functions to design, register, modify, and remove workflow definitions for workflow administrators. For workflow processors, it provides functions to initiate, pull back, cancel, approve, reject, and reference workflow transactions. For system administrators, it provides functions to maintain the system including user management. The system also provides functions to audit the record for auditors. The benefit of the workflow management system is that it improves the efficiency and reduces the cost while complying with internal regulations.

\subsection{Assumption of Cross Organizational Workflow}

When the workflow management system is expanded to cross organizational operation, the steps in workflow definition are processed by users from multiple organizations. In this paper, a cross organizational workflow is assumed as a combination of internal and external workflows (Figure 2).

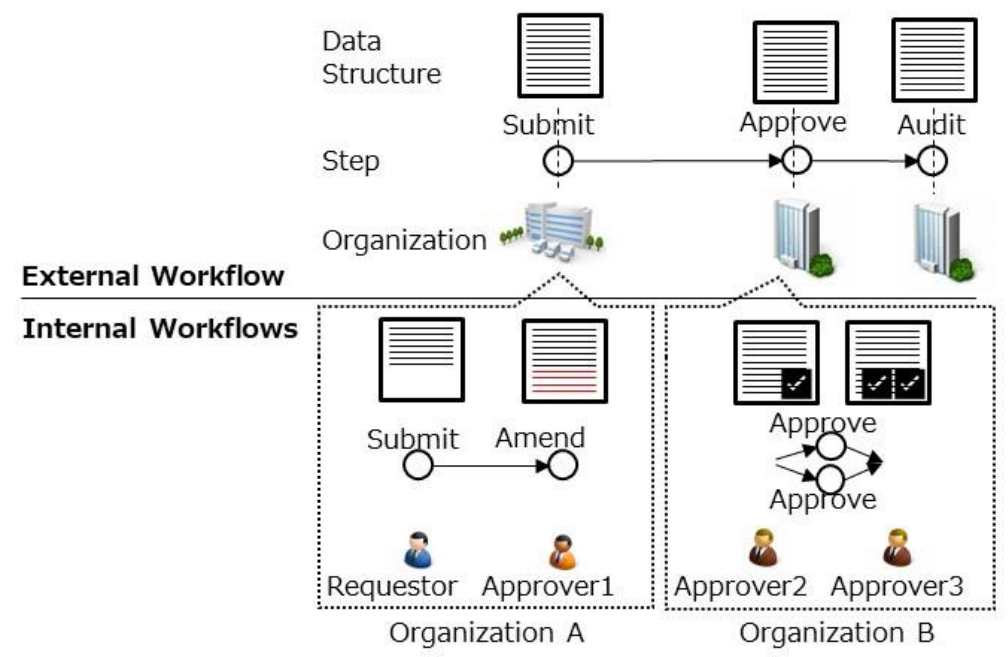

Figure 2. Overview of cross organizational workflow

The internal workflow is managed in the same way as the internal workflow management system defined in Section 2.1. However, the external workflow should be managed in a different manner. It should be defined by workflow administrators from multiple organizations based on an agreement between organizations, processed by processors from multiple organizations, and audited by third party auditor.

\subsection{Requirement for Cross Organizational Workflow Management System}

As described in section 2.2, the external workflow in a cross organizational workflow is defined, processed, and managed between organizations which do not fully trust each other. Therefore, the system needs to be designed to accommodate some additional feature in order to securely process workflow among trustless organizations.

There are two additional requirements for a cross organizational workflow management system. One is to secure that the workflow is processed as agreed among the organizations beforehand. The other is to store and provide immutable audit trails of transactions which include the set of proposed data and the approvers. 
However, if the cross organizational workflow management system is implemented as a centralized system, there are three falsification risks as shown in Table 3.

Table 3. Falsification risks on a workflow management system

\begin{tabular}{lll}
\hline Target & Risk & Caused-by \\
\hline Workflow Definition & Alter Definition & Workflow Administrator \\
Workflow State & Skip step & System Administrator \\
& Alter State & System Administrator \\
Transaction Record & Alter Record & System Administrator \\
\hline
\end{tabular}

These falsification risks are caused by two SPoT in the system. One is the workflow administrator. If the workflow definition is managed by a single responsible person in the system, the person can falsify the definition on their own decision. The other is system administrator. If the system is managed by a single responsible administrator, they can falsify the state of workflow or the proposal data (Figure 3).

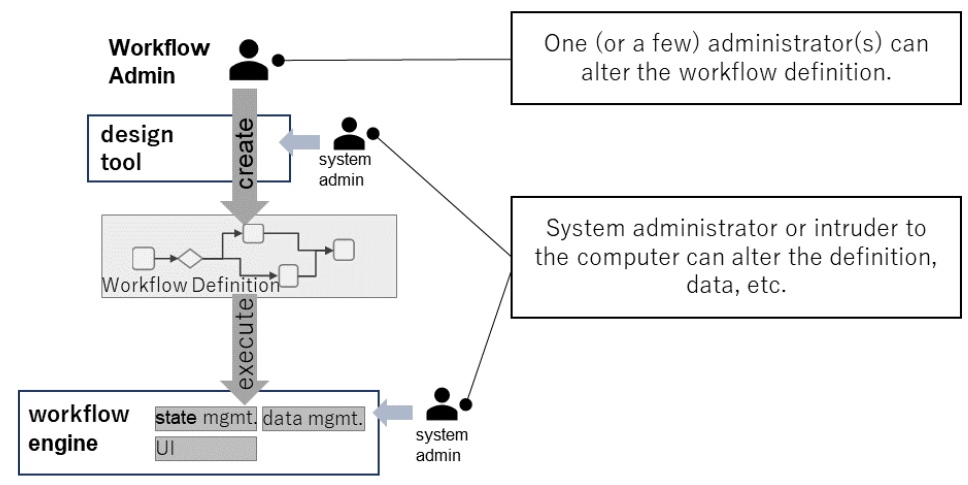

Figure 3. Single Point of Trust in Workflow management system

The cross organizational workflow management system should satisfy these two additional requirements described above by eliminating the two SPoT in order to avoid the three falsification risks.

\section{OUTLINE OF CROSS ORGANIZATIONAL WORKFLOW MANAGEMENT SYSTEM}

\subsection{System Architecture}

Figure 4 depicts the proposed system architecture in which the two SPoT described in section 2.3 are eliminated utilizing consortium blockchain with smart contract function. Consortium blockchain is one of the types of blockchain where multiple organizations govern the network. In this architecture, it is assumed that each organization which processes the workflow owns the blockchain node and each node accommodates two types of smart contract. One is to manage workflow definition and the other is to manage workflow processing. The smart contract to manage workflow definition accept requests from workflow administrators of each organization through the workflow admin web application. This smart contract manages the requests for workflow definition from the viewpoint of each organization, such as requirements for data items, steps, and approvers. The request is sent to other nodes and the smart contract on each node validates the data format and the user privilege and checks conflicts between requests. If the result of this validation is the same as each other, then the smart contract writes the result into the workflow definition store on each node. Thus, workflow definitions are organized by the smart contract based on the requests from the workflow administrators and it is impossible to alter the definitions in all nodes by one of the workflow administrators. The first SPoT of workflow administrator is thus eliminated by this smart contract. 
The other smart contract is to manage workflow processing. This smart contract processes the workflow based on the workflow definition that an initiator indicates in the first step. Each request to process workflow is accepted by the smart contract for workflow processing and it validates the results from each of the nodes and writes the result into workflow state database on each node. In this architecture, the system administrator of each organization manages their own node. They can alter the state of workflow in their own node, but as described above, the smart contract validates the result in each node and if the results are different from the one by other node, it will be rejected, and the user can be aware of the falsification. Thus, the second SPoT of system administrator is eliminated.

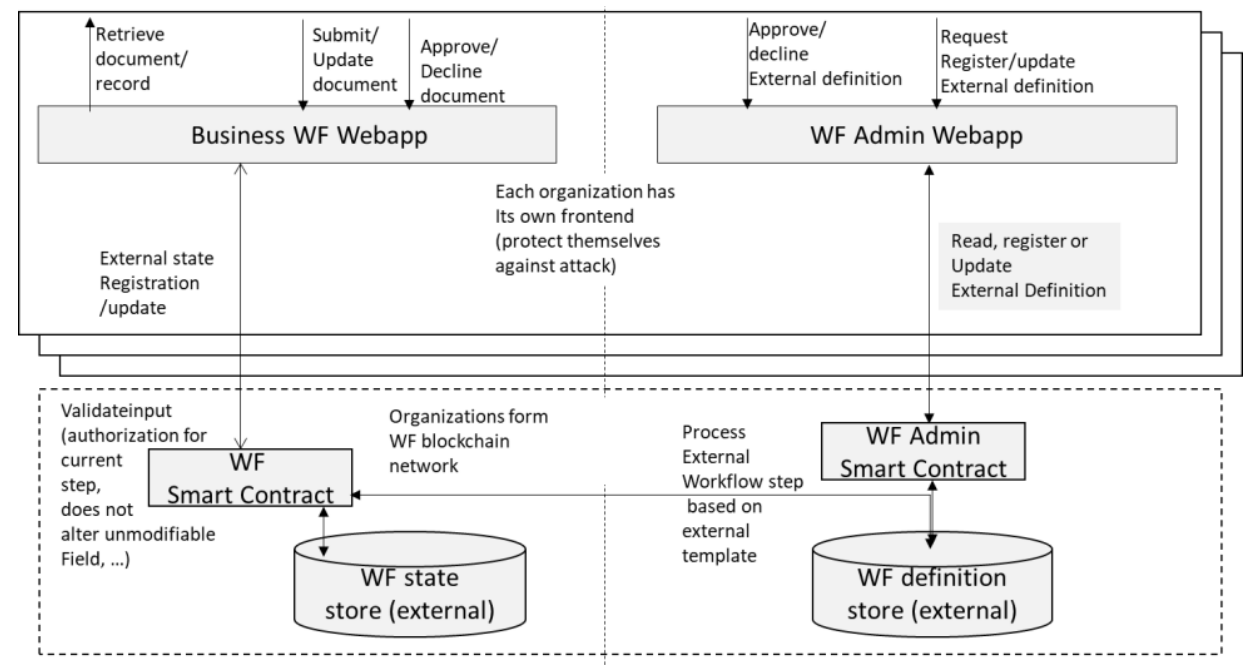

Figure 4. System architecture of Blockchain based cross organizational workflow management system

\subsection{Transaction Flow}

This section describes how a workflow transaction is processed and the data is stored while eliminating SPoT by the architecture described in section 3.1. Figure 5 shows the transaction flow when a workflow processor submits a transaction to initiate a workflow. First the web application on the blockchain node in one of the participating organizations shows the list of workflow templates that the processor can initiate. The processor selects one of the templates, set required values and submit the proposal. Then the smart contract on the node distributes the proposal to other nodes and each node validates the proposal including the user authority, processes the code for proposal and creates a read/write set to be stored in state database. This read/write set includes the proposal data itself and the result of the processed code. If the returned read/write set from other nodes are the same as the result of this node, then the node requests other nodes to write the result. In the state database, the workflow proposal is stored with the request ID and the action which is created in the smart contract as the next step to be processed by an approver or a reviewer.

Figure 6 shows the transaction flow of the reference process. In case a user requests to refer a record to know the status of a proposal or to audit a proposal after the case is closed, the smart contract processes the request in the same way as a processing request as described above. And the smart contract on each node write the result of the reference request into the state database in order to verify that the results among state databases on each node are consistent through the validation process by smart contract. Thus, the transaction flow of the proposed system architecture keeps the consistency of the stored record even after a case is closed. 


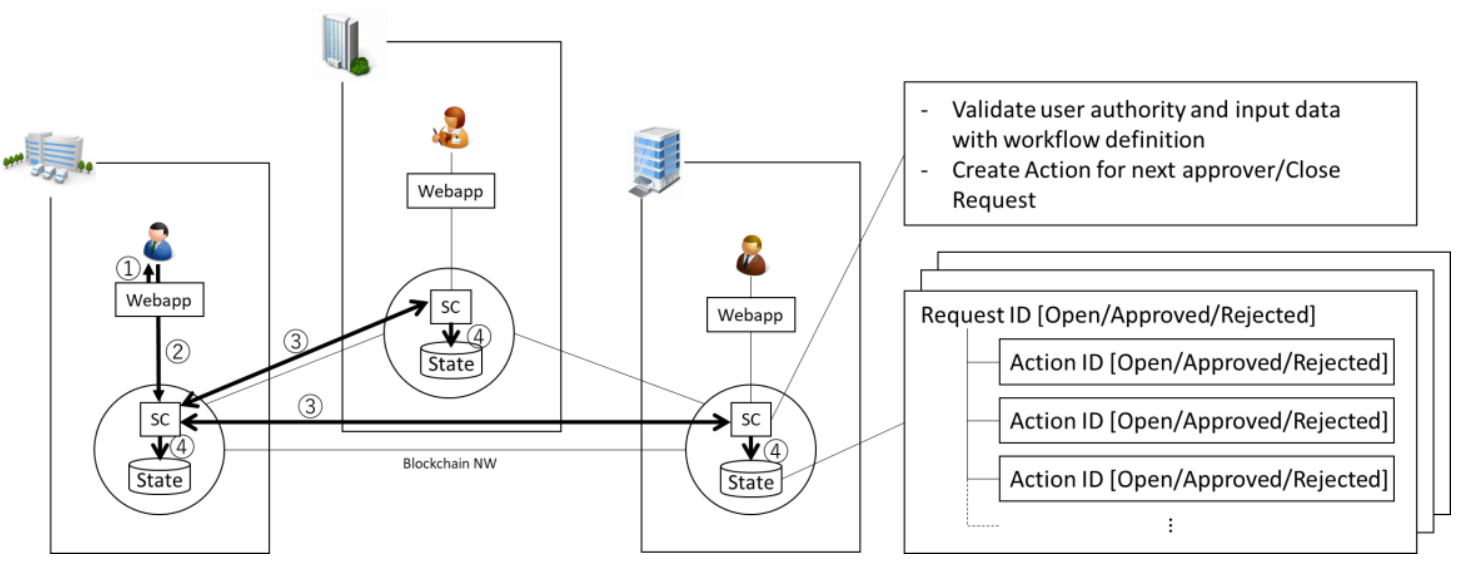

Figure 5. Transaction flow of create/update process

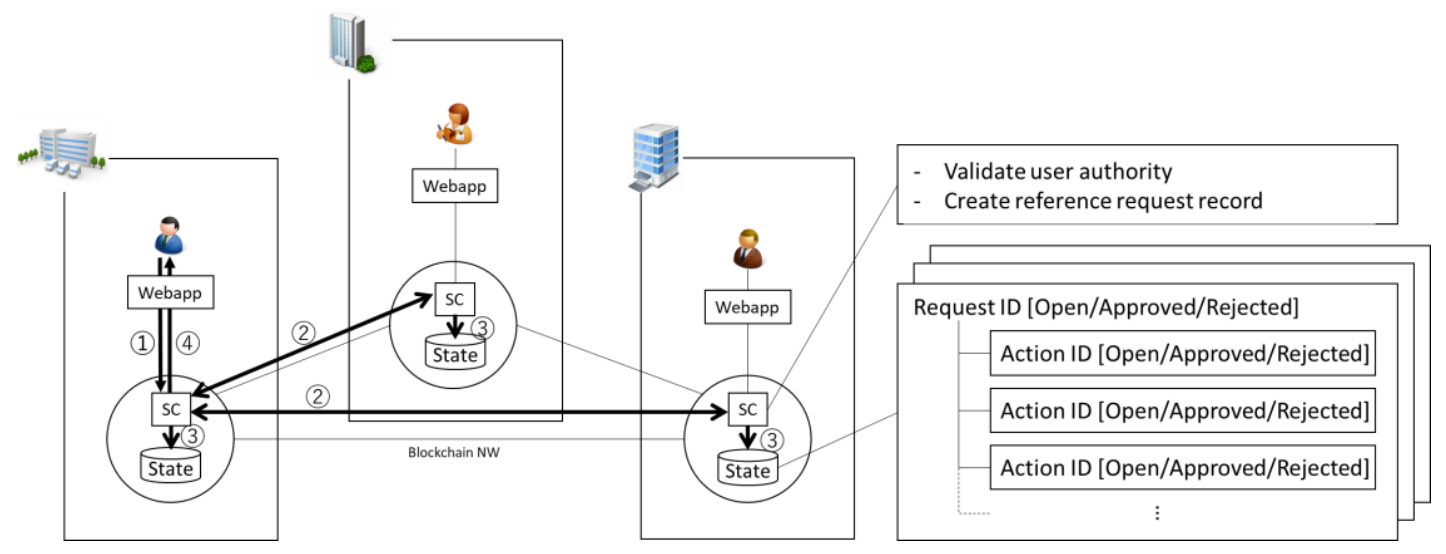

Figure 6. Transaction flow of reference process

\section{IMPLEMENTATION AND EVALUATION OF PROPOSED SYSTEM ARCHITECTURE}

\subsection{Implementation of Cross Organizational Workflow Use Case}

As described in (van der Aalst, et al., 2003), there are many patterns of workflow structure and control, but all the patterns are composed by the combination of proposing, approving, reviewing, and rejecting transactions. In order to demonstrate the feasibility of the transaction flow on the proposed system architecture, a simple use case which processes a cross organizational workflow among three organizations for customer onboarding (Figure 7) is implemented as a workflow smart contract as described in section 3.1. This use case is implemented on Hyperledger Fabric (The Linux Foundation, online) which is one of the major implementations of the consortium blockchain as a platform to implement the smart contract. In this use case, the requestor in one of the organizations submits a proposal with related information and then other organizations approve or reject the proposal in sequence. Each action they take is stored in the state database as the execution result of the smart contract, and the state of designated workflow is updated. 


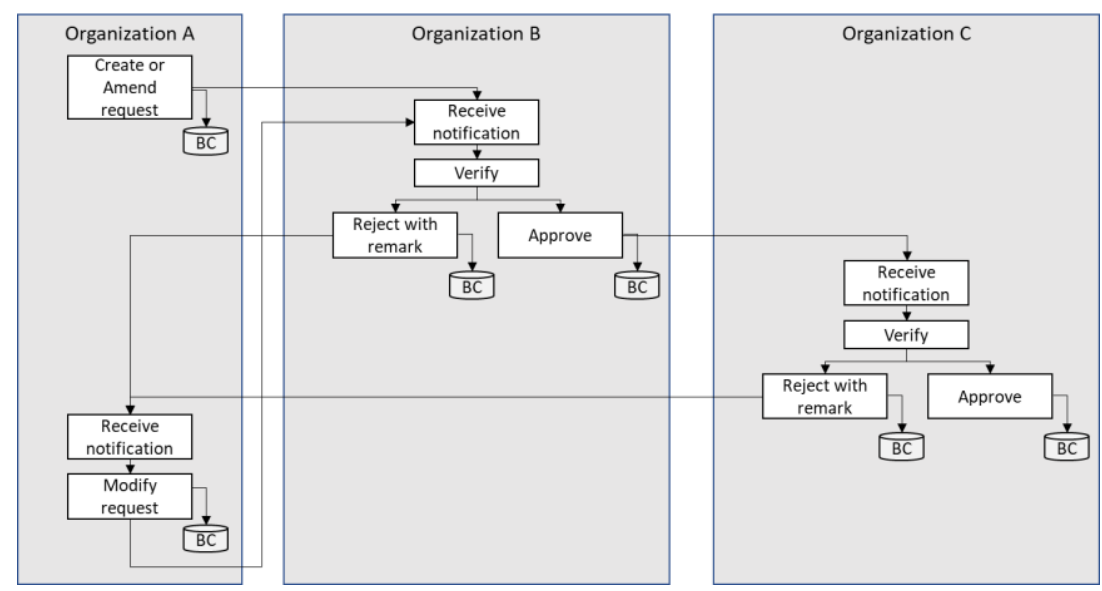

Figure 7. Use case of implemented workflow

Each action is processed by the workflow smart contract as described in section 3.2. The workflow smart contract validates the input values based on the workflow definition and if it is passed, it creates a read/write set to store in the state database as a new state with the inputs. Then the approver logs in to this system and the web application retrieves and shows the list of cases which are assigned to this approver based on the workflow status. The approver selects one of the cases from the list, checks the values and then approves or rejects the proposal. The smart contract validates the signature and the value and if it is passed, it creates a $\mathrm{read} / \mathrm{write}$ set in the same way as the request processing. Every action of request, approval and rejection is recorded in the state database as depicted in Figure 5 and Figure 6. Even if one of the system administrator alters the data on their own node, it is not reflected to other nodes and it can be found when the next transaction is processed by the smart contract because the organizations cannot agree on the result for the next transaction.

\subsection{Security Evaluation of Proposed System Architecture}

To evaluate the proposed system architecture from the viewpoint of falsification risk, the attack scenarios based on workflow lifecycle as shown in Figure 8 are assumed. This section describes how the proposed system architecture avoids the falsification risk in each scenario.

Agreement

\begin{tabular}{|c|c|c|}
\hline of workflow definition & \multicolumn{2}{|c|}{ Close case } \\
\hline $\begin{array}{l}\text { (1) Falsification of workflow definition } \\
\text { by workflow Administrator or system } \\
\text { Administrator }\end{array}$ & $\begin{array}{l}\text { (2) Falsification of workflow definition } \\
\text { by workflow administrator or system } \\
\text { administrator } \\
\text { (3) Falsification of proposal data by } \\
\text { system administrator }\end{array}$ & $\begin{array}{l}\text { (4) Falsification of proposal data } \\
\text { by system administrator }\end{array}$ \\
\hline
\end{tabular}

Figure 8. Attack scenarios in workflow lifecycle

(1) The first scenario is the falsification of workflow definition by one of the workflow administrators among participating organizations before opening a case. They may falsify the definition to skip the step of approval. In the proposed system architecture, the workflow definition is implemented as a smart contract and it is distributed into every processing node. And the smart contracts send the $\mathrm{read} /$ write set of the transaction each other to be processed in other nodes. If one of the smart contracts on the processing node is falsified by the administrator, the validation does not pass, and the transaction will be rejected. Thus, the first falsification risk is avoided in the proposed system architecture.

(2) The second scenario is the falsification of workflow definition by one of the workflow administrators among participating organizations during a case is open. The motivation of malicious 
user is the same as the first scenario and this is avoided as same as the first scenario in the proposed system architecture.

(3) The third scenario is the falsification of the proposal data by a system administrator while a case is open. They may falsify the data after another administrator approved the proposal. In the proposed system architecture, each node executes the smart contract and validate the results each other. If the proposal data in one of the nodes is falsified, the result will be different from the result of other nodes and the validation fails. Thus, this risk is avoided in the proposed system architecture.

(4) Fourth scenario is the falsification of proposal data by a system administrator after a case is closed. They may falsify the data after all the approvers approved the case. In the proposed system architecture, each node executes the smart contract and validate the results each other even if this case is already closed and it is just a reference transaction. Thus, this falsification risk is also avoided in the proposed system architecture.

Thus, our proposed system architecture avoids all the falsification risks by eliminating the SPoT in cross organizational workflow management system.

\section{CONCLUSION}

In this paper, the blockchain-based cross organizational workflow management system among trustless organizations is proposed and the smart contract for workflow processing among organizations is implemented. Through the evaluation of falsification risks based on the attack scenarios along the workflow lifecycle, it is confirmed that our proposed system architecture avoids the falsification risks by eliminating the SPoT among organizations and provide efficient and quicker workflow processing for enterprise use.

\section{REFERENCES}

Alves, P. et al, 2020. Exploring Blockchain Technology to Improve Multi-party Relationship in Business Process Management Systems. ICEIS 2020 - 22nd International Conference on Enterprise Information System.

Fridgen, G. et al, 2018. Cross-Organizational Workflow Management Using Blockchain Technology - Towards Applicability, Auditability, and Automation. Proceedings of the 51st Hawaii International Conference on System Sciences. Hawaii, USA, pp. 3507-3516

Hileman, G. \& Rauchs, M., 2017. 2017 Global Blockchain Benchmarking Study. [Online] Available at: https://ssrn.com/abstract=3040224 (last visited Oct. 12, 2020)

Kumar, A. \& Zhao, J.L., 2002. Workflow support for electronic commerce applications. Decision support systems, Vol. 32, No. 3, pp. 265-278.

Nakamoto, S., 2008. Bitcoin: A Peer-to-Peer Electronic Cash System. [Online] Available at: https://bitcoin.org/bitcoin.pdf (last visited Oct. 12, 2020)

Reijers, H. et al, 2016. The effectiveness of workflow management systems: A longitudinal study. International Journal of Information Management, Vol.36, pp 126-141.

Stohr, A. E. \& Zhao, J.L., 2001. Workflow Automation: Overview and Research Issues. Information Systems Frontiers, Vol. 3 No. 3, pp 281-296.

The Linux Foundation, online. Hyperledger Fabric - Hyperledger. [Online] Available at: https://www.hyperledger.org/use/fabric (last visited Oct. 12, 2020)

van der Aalst, W. et al, 2016. Business Process Management Don't Forget to Improve the Process!. Business \& Information Systems Engineering, Vol. 58, No. 1, pp 1-6.

van der Aalst, W. et al, 2003. Workflow Patterns. Distributed and Parallel Databases, Vol. 14, No. 1, pp 5-51.

Weber, I. et al, 2016. Untrusted Business Process Monitoring and Execution Using Blockchain., International Conference on Business Process Management., s.l.

World Economic Forum, 2018. Trade Tech - A New Age for Trade and Supply Chain Finance. [Online] Available at: http://www3.weforum.org/docs/White_Paper_Trade_Tech_report_2018.pdf (last visited Oct. 12, 2020)

World Trade Organization, 2018. Can Blockchain revolutionize international trade?. [Online] Available at: https://www.wto.org/English/res_e/booksp_e/blockchainrev18_e.pdf (last visited Oct. 12, 2020) 